\title{
The Effect of Food on Tramadol and Celecoxib Bioavailability Following Oral Administration of Co-Crystal of Tramadol-Celecoxib (CTC): A Randomised, Open-Label, Single-Dose, Crossover Study in Healthy Volunteers
}

\author{
Gregorio Encina ${ }^{1}\left(\right.$ Mercedes Encabo $^{1} \oplus \cdot$ Marisol Escriche $^{1}\left(\right.$ Mounia Lahjou $^{2,3} \cdot$ Eric Sicard $^{4} \cdot$ Kevin Smith $^{5}$.

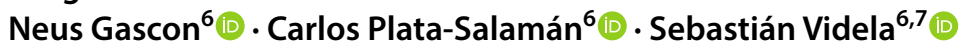

Published online: 14 July 2018

(c) The Author(s) 2018

\begin{abstract}
Background and Objective Co-Crystal of Tramadol-Celecoxib (CTC), in development for the treatment of moderate to severe acute pain, is a first-in-class co-crystal containing a 1:1 molecular ratio of two active pharmaceutical ingredients; ractramadol $\cdot \mathrm{HCl}$ and celecoxib. This randomised, open-label, crossover study compared the bioavailability of both components after CTC administration under fed and fasting conditions.

Methods Healthy adults received single doses of $200 \mathrm{mg}$ CTC under both fed and fasting conditions (separated by a 7-day washout). Each dose of CTC was administered orally as two $100 \mathrm{mg}$ tablets, each containing $44 \mathrm{mg}$ tramadol. $\mathrm{HCl}$ and $56 \mathrm{mg}$ celecoxib. In the fed condition, a high-fat, high-calorie meal [in line with recommendations by the US Food and Drug Administration (FDA)] was served 30 min before CTC administration. Tramadol, $O$-desmethyltramadol and celecoxib plasma concentrations were measured pre- and post-dose up to $48 \mathrm{~h}$. Pharmacokinetic parameters were calculated using non-compartmental analysis. Safety was also assessed.

Results Thirty-six subjects (18 female/18 male) received one or both doses of CTC; 33 provided evaluable pharmacokinetic data under fed and fasting conditions. For tramadol and $O$-desmethyltramadol, fed-to-fasting ratios of geometric least-squares means and corresponding $90 \%$ confidence interval $(\mathrm{CI})$ values for maximum plasma concentration $\left(C_{\max }\right)$ and extrapolated area under the plasma concentration-time curve to infinity $\left(\mathrm{AUC}_{\infty}\right)$ were within the pre-defined range for comparative bioavailability (80-125\%). For celecoxib, $C_{\max }$ and $\mathrm{AUC}_{\infty}$ fed-to-fasting ratios $(90 \% \mathrm{CIs}$ ) were outside this range, at $130.91 \%$ (116.98-146.49) and $129.34 \%$ (121.78-137.38), respectively. The safety profile of CTC was similar in fed and fasting conditions.

Conclusions As reported for standard-formulation celecoxib, food increased the bioavailability of celecoxib from single-dose CTC. Food had no effect on tramadol or $O$-desmethyltramadol bioavailability.

Clinical trial registration number 152052 (registered with the Therapeutic Products Directorate of Health Canada)
\end{abstract}

Electronic supplementary material The online version of this article (https://doi.org/10.1007/s40261-018-0672-y) contains supplementary material, which is available to authorized users.

Gregorio Encina

gencina@esteve.com

Extended author information available on the last page of the article 


\section{Key Points}

Co-Crystal of Tramadol-Celecoxib (CTC), a first-inclass active pharmaceutical ingredient (API-API) cocrystal of rac-tramadol $\cdot \mathrm{HCl}$ and celecoxib, is in development for the treatment of moderate to severe acute pain.

Food effects are reported for standard drug formulations of celecoxib; the present study investigated the effects of food on the oral bioavailability of tramadol and celecoxib after CTC (200 mg) administration.

As for standard drug formulations of the individual reference products, food increased the bioavailability of celecoxib but not that of tramadol; therefore, as for these reference products, specific dosing recommendations relating to meal times are not anticipated to be required for CTC.

\section{Introduction}

Pharmaceutical co-crystals, which usually consist of an active pharmaceutical ingredient (API) and one or more coformers, provide a means to modify the physicochemical, pharmacokinetic - and therefore potentially the clinicalproperties of the constituent API [1-3]. Co-crystals containing more than one API (multidrug or 'API-API' co-crystals) may also provide a novel method of delivering a drug treatment with multiple mechanisms of action. Co-Crystal of Tramadol-Celecoxib (CTC) is a first-in-class API-API co-crystal in development for the treatment of moderate to severe acute pain.

CTC contains rac-tramadol hydrochloride $(\mathrm{HCl})$ and celecoxib in a 1:1 molecular ratio, with $100 \mathrm{mg}$ CTC comprising $44 \mathrm{mg}$ tramadol. $\mathrm{HCl}$ and $56 \mathrm{mg}$ celecoxib. This co-crystal targets pain through four complementary mechanisms of action: cyclooxygenase- 2 inhibition, $\mu$-opioid receptor agonism, noradrenaline reuptake inhibition and serotonin reuptake inhibition [4]. CTC may therefore provide multimodal analgesia, a treatment approach recommended in acute pain [5, 6], the optimal management of which continues to be hampered by lack of treatment efficacy [5, 7] and issues regarding side effects [8]. Analytical studies have confirmed that the physicochemical properties of both tramadol and celecoxib are modified by co-crystallisation [4]. For example, the intrinsic dissolution rate of tramadol is slower, and that of celecoxib faster, compared with individual reference products [4]. Synergistic antinociceptive effects have been observed in preclinical in vivo studies, without potentiation of adverse effects [9]. The analgesic effects of CTC (100, 150 and
$200 \mathrm{mg}$ ) have also been demonstrated in a Phase 2 clinical study in patients with acute moderate to severe pain following extraction of two or more impacted third molars requiring bone removal [10].

The clinical pharmacokinetics of CTC were first characterised in two Phase 1 studies conducted in healthy male and female subjects; one assessing a single dose of CTC [11] and one assessing multiple doses [12]. In both studies, differences in the pharmacokinetics of tramadol and celecoxib were seen after administration of CTC compared with after administration of the individual reference products (immediate-release tramadol or celecoxib) alone or in free combination. In the case of tramadol, a lower maximum observed plasma concentration $\left(C_{\max }\right)$, slightly prolonged time to $C_{\max }\left(T_{\max }\right)$ and similar area under the plasma concentration-time curve (AUC) were observed with CTC. In the case of celecoxib pharmacokinetics after CTC administration, celecoxib had a lower $C_{\text {max }}$ compared with celecoxib alone, and a faster $T_{\max }$ compared with celecoxib alone or in free combination with tramadol [11, 12].

The Phase 1 studies described above were conducted in fasting conditions. The bioavailability of tramadol from its standard drug formulation is not affected by food intake [13]. However, a high-fat meal slows absorption of celecoxib from its standard formulation by around $1 \mathrm{~h}$, and increases the $C_{\max }$ and AUC $[14,15]$. The primary objective of the present study was therefore to assess the bioavailability of tramadol and celecoxib after a single dose of CTC, when administered in the fed or fasting state. The secondary objective was to evaluate the safety of CTC following single-dose administration in the fed or fasting state.

\section{Methods}

\subsection{Study Subjects}

Subjects were male or female volunteers aged 18-55 years, with a body mass index $\geq 18.5$ and $<29 \mathrm{~kg} / \mathrm{m}^{2}$. Subjects were required to be in good health, with no clinically significant findings as determined by physical examination, electrocardiogram (ECG), clinical laboratory evaluations (haematology, biochemistry and urinalysis) and review of the subject's medical history. Subjects were also required to be non-smokers (or ex-smokers for at least 6 months). Key exclusion criteria included pregnancy or lactation; a history of significant hypersensitivity to tramadol, celecoxib, sulphonamides, opioid analgesics or any related products; or a history of severe hypersensitivity reactions to any drugs. Full inclusion and exclusion criteria are included in Online Resource 1. 


\subsection{Study Design and Treatments}

This study was a single-centre, randomised, single-dose, open-label, two-period, two-sequence, crossover, food effect study. The study was conducted by a clinical research organisation (CRO; Algorithme Pharma) at a single site in Quebec, Canada and registered with the Therapeutic Products Directorate of Health Canada (152052). The study protocol was approved by an independent ethics committee and the study was performed in compliance with Good Clinical Practice. Institutional review board guidelines on the treatment of human subjects were followed, meeting the requirements of the Declaration of Helsinki. All subjects voluntarily enrolled in the study and gave written informed consent.

Each subject received a single oral dose of $200 \mathrm{mg}$ CTC, once under fed conditions and once under fasting conditions. This dose had previously been evaluated in two earlier Phase 1 studies $[11,12]$ and was also efficacious and well tolerated in a Phase 2 study in which doses of 50, 100, 150 and $200 \mathrm{mg}$ CTC were administered [10]. CTC (Esteve Pharmaceuticals, S.A., Spain) was provided as two $100 \mathrm{mg}$ tablets, each containing $44 \mathrm{mg}$ tramadol $\cdot \mathrm{HCl}$ and $56 \mathrm{mg}$ celecoxib. Subjects were randomly assigned to one of two sequences-fed-fasting or fasting-fed-via a list generated using a computer application developed by Algorithme Pharma. Dosing was separated by a 7-day washout period (Fig. 1). All subjects underwent a supervised overnight fast ( $\geq 10 \mathrm{~h}$ ) before each CTC administration and fasted for at least $4 \mathrm{~h}$ following administration, after which a standardised meal was served. In the fed condition, the overnight fast was followed by consumption of a standardised high-fat, high-calorie meal [in line with the US Food and Drug Administration (FDA) recommendations] [16] 30 min before CTC administration. During the study, restrictions were applied to prescription medications; over-the-counter products; alcohol; xanthine, grapefruit- and pomelo-containing food or beverages; and strenuous activity. Water was permitted ad libitum until 1-h pre-CTC administration and again from 1 -h postadministration. CTC was administered with approximately $240 \mathrm{~mL}$ water.

\subsection{Pharmacokinetic Sampling and Analytical Methods}

During each treatment period, blood samples ( $4 \mathrm{~mL}$ each) were collected for pharmacokinetic analysis before CTC administration (at $0 \mathrm{~h}$ ) and at $0.5,1,1.5,1.75,2,2.33,2.67$, $3,3.5,4,5,6,8,10,12,16,24,36$ and 48 h post-administration. Additional samples were taken at $3.33,3.67,4.5$ and $5.5 \mathrm{~h}$ post-administration in the fed state and at 0.75 and $1.25 \mathrm{~h}$ post-administration in the fasting state. Samples were collected in pre-cooled $\mathrm{K}_{2}$-EDTA-containing tubes and centrifuged (at $4{ }^{\circ} \mathrm{C}$ and $1500 \times g$ for $10 \mathrm{~min}$ ) as soon as possible after collection. Plasma was then separated, frozen at a nominal temperature of $-20{ }^{\circ} \mathrm{C}$ and transported on dry ice to the laboratory for assay.
Fig. 1 Study design and subject disposition. *The terminal phases of celecoxib could not be adequately estimated following CTC administration in the fasting state in three subjects. $A U C_{\infty}$ extrapolated area under the plasma concentration-time curve to infinity, CTC Co-Crystal of Tramadol-Celecoxib, $P K$ pharmacokinetic, $T_{1 / 2 e l}$ elimination half-life

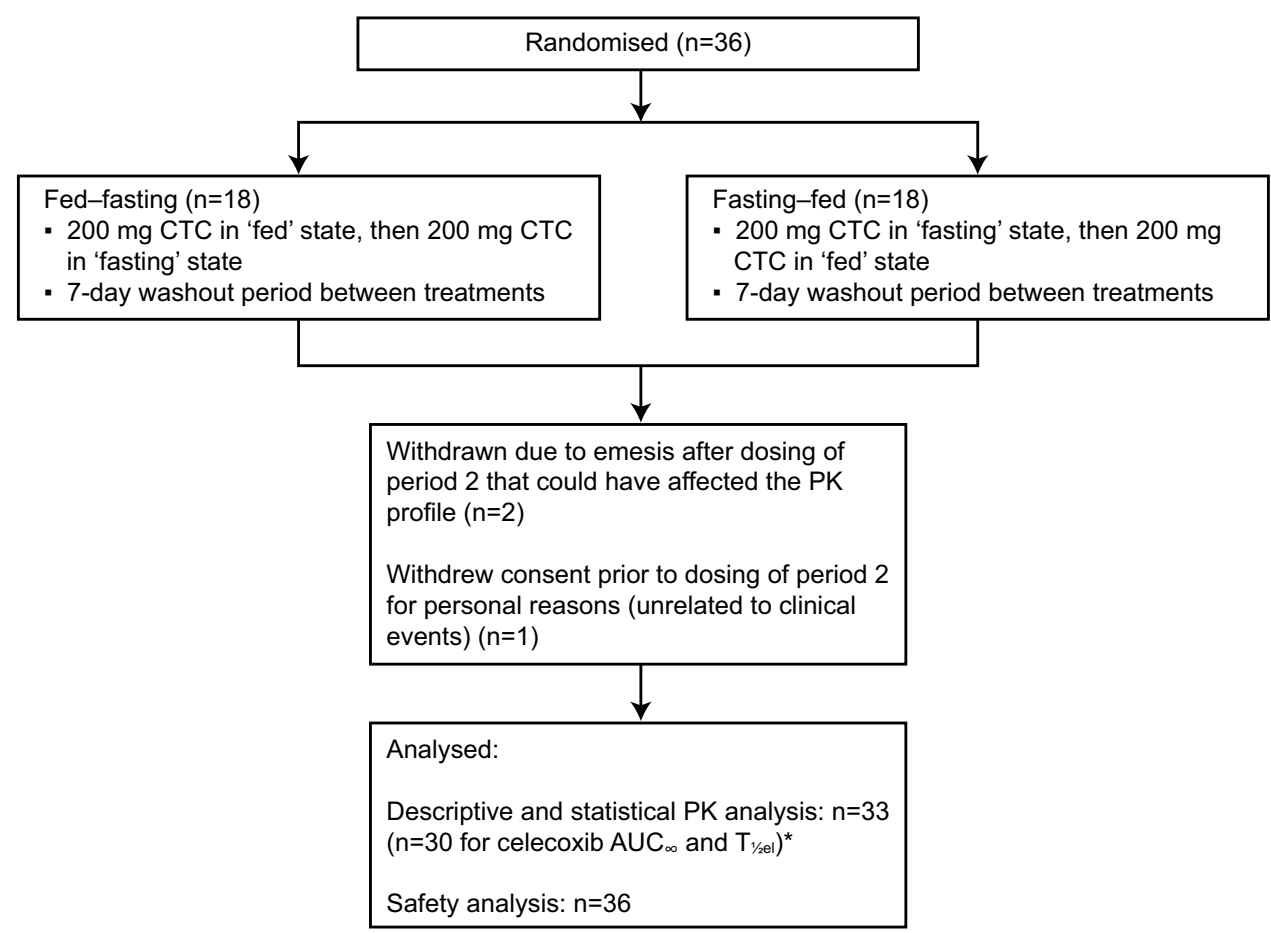




\subsection{Bioanalytical Assay}

Plasma concentrations of tramadol, $O$-desmethyltramadol (tramadol's main metabolite) and celecoxib were measured using validated high-performance liquid chromatography with tandem mass spectrometry methods.

Samples $(0.05 \mathrm{~mL})$ were extracted by solid-phase extraction in Oasis MCX 96-well plates (10 mg sorbent, $30 \mu \mathrm{m}$ particle size; Waters Corporation). After clean-up, the eluate obtained with methanol was used for analysis of celecoxib and the posterior eluate obtained with 5\% $\mathrm{NH}_{4} \mathrm{OH}$ in methanol was used for analysis of tramadol and $O$-desmethyltramadol. Celecoxib was separated on an XBridge C18 column $(3.5 \mu \mathrm{m} 50 \times 2.1 \mathrm{~mm}$; Waters Corporation) and tramadol and $O$-desmethyltramadol were separated on an Acquity UPLC BEH Shield RP18 column $(1.7 \mu \mathrm{m} 2.1 \times 50 \mathrm{~mm}$; Waters Corporation) using different gradients of methanol and $0.1 \%$ formic acid in water. Detection for celecoxib was performed via tandem mass spectrometry on an API 4000 triple quadrupole mass spectrometer (Sciex, Ontario, Canada) using negative electrospray ionisation. For tramadol and $O$-desmethyltramadol, a Xevo TQ MS triple quadrupole mass spectrometer using positive electrospray ionisation (Waters Corporation) was used. Propranolol and E-6087, a cyclooxygenase- 2 inhibitor, were used as internal standards for tramadol (and $O$-desmethyltramadol) and celecoxib, respectively.

During validation of these methods, parameters evaluated were linearity and range; selectivity; specificity; intra- and inter-run precision and accuracy; limit of quantification; dilution integrity; carry over; recovery; matrix effect; and stability in the autosampler at room temperature, after freeze-thaw cycles and over the long term. The lower and upper limits of quantification were 4.00 and $640.00,1.00$ and 160.00 , and 2.50 and $1000.00 \mathrm{ng} / \mathrm{mL}$, for tramadol, $O$-desmethyltramadol and celecoxib, respectively. Assay inter-run precision (coefficient of variation; $\mathrm{CV}$ ) and accuracy (nominal values) for the limit of quantification were 8.3 and $102.5 \%$, respectively for tramadol, 10.1 and $105.2 \%$ for $O$-desmethyltramadol and 10.5 and $107.8 \%$ for celecoxib. Assay specificity was assessed using six independent matrix sources, verified for the absence of interference, and compared with the respective limits of quantification at the retention times and mass transitions of analytes and internal standards. The absolute recovery ranged from 70.2 to $73.3 \%$ for tramadol, $76.3-80.5 \%$ for $O$-desmethyltramadol and $81.6-86.2 \%$ for celecoxib. Samples were stored for a maximum of 63 days (the long-term stability of frozen samples was confirmed up to 154 days).

\subsection{Safety Assessments}

Safety was monitored throughout the study via the assessment of adverse events (AEs), classified using the Medical Dictionary for Regulatory Activities (MedDRA), version 13.1. Assessment of vital signs and standard clinical laboratory parameters (haematology, biochemistry and urinalysis), ECGs and a physical examination were conducted at screening and at the end of the study, and vital signs were also measured prior to each CTC administration.

\subsection{Data Analyses and Statistics}

Findings from previous studies conducted with celecoxib at the CRO suggested that the intra-subject CV following a single dose of celecoxib was approximately $28 \%$ for $C_{\max }$ and around $12 \%$ for cumulative AUC from time zero to time of last observed quantifiable plasma concentration $\left(\mathrm{AUC}_{t}\right)$. Ratios of geometric least-squares (LS) means were expected to fall between 95 and $105 \%$. Therefore, it was estimated that 32 subjects would be required to provide an adequate assessment of the effect of food on celecoxib bioavailability following CTC administration. Thirty-six subjects were recruited to allow for the possibility of drop-outs and variations around the estimated intra-subject $\mathrm{CV}$. As celecoxib was known to be subject to higher intra-subject variability than tramadol or $O$-desmethyltramadol, these calculations also provided an adequate estimation of the sample size required to assess the bioavailability of these latter two analytes.

The main pharmacokinetic parameters evaluated for all three analytes were $C_{\max } ; T_{\max } ; \mathrm{AUC}_{i}$; extrapolated AUC to infinity $\left(\mathrm{AUC}_{\infty}\right)$; and elimination half-life $\left(T_{1 / 2 \mathrm{el}}\right)$. Descriptive pharmacokinetic analyses were conducted using a noncompartmental approach and a log-linear terminal phase was assumed. The trapezoidal rule was used for AUC estimates and the terminal phase was estimated by maximizing the coefficient of determination calculated using a log-linear regression model. The natural logarithmic (ln) transformations of $C_{\max }, \mathrm{AUC}_{t}$ and $\mathrm{AUC}_{\infty}$ were used for statistical pharmacokinetic analyses. All pharmacokinetic parameters except $T_{\max }$ were analysed using an analysis of variance (ANOVA; in line with regulatory requirements [17]), with fixed factors of subject (nested within sequence), treatment, treatment period and sequence. $T_{\max }$ was analysed via a non-parametric approach, using Wilcoxon's rank sum test (Mann-Whitney $U$ test). The comparative bioavailability of tramadol, M1 and celecoxib between fed and fasting states was assessed using a confidence interval (CI) approach. Fedto-fasting ratios of geometric LS means and corresponding $90 \%$ CIs were calculated for $C_{\max }, \mathrm{AUC}_{t}$ and $\mathrm{AUC}_{\infty}$. For each analyte, bioavailability was considered comparable if 
fed-to-fasting ratios and corresponding 90\% CIs of these parameters were within the pre-defined range of $80-125 \%$.

Subjects who provided measurable data in both treatment conditions were included in the descriptive and statistical pharmacokinetic analyses. All subjects who received at least one dose of CTC were included in the safety analyses.

\section{Results}

\subsection{Subjects}

The study was initiated on 25 January 2012 and completed on 8 February 2012. Thirty-six subjects (18 male and 18 female) were enrolled and received at least one dose of CTC; 35 of these subjects were administered single-dose CTC in both fed and fasting conditions. Subject demographics and characteristics are summarised in Table 1. Thirty-four

Table 1 Subject demographics and other characteristics $(N=36)$

\begin{tabular}{lc}
\hline Characteristic & \multicolumn{1}{l}{ Value } \\
\hline Age, years; mean (range) & $35(18-54)$ \\
Gender; $n(\%)$ & \\
Male & $18(50.0)$ \\
Female & $18(50.0)$ \\
Race; $n(\%)$ & \\
White & $34(94.4)$ \\
Asian & $2(5.6)$ \\
Weight, kg; mean (SD) & $65.7(10.9)$ \\
Height, cm; mean (SD) & $167.3(10.5)$ \\
Body mass index, $\mathrm{kg} / \mathrm{m}^{2} ;$ mean $(\mathrm{SD})$ & $23.4(2.5)$ \\
\hline
\end{tabular}

$S D$ standard deviation subjects (94\%) were white; mean age was 35 years. One subject withdrew consent prior to dosing in the fasting state for personal reasons that were unrelated to any clinical event. Two subjects were withdrawn after dosing in the fed state due to emesis that could have affected pharmacokinetic findings. All subjects were included in the safety analysis. Thirty-three subjects provided evaluable pharmacokinetic data in both fed and fasting conditions and were therefore included in both descriptive and statistical pharmacokinetic analyses (Fig. 1). In a further three subjects, the terminal phases of celecoxib could not be adequately estimated following administration of CTC in the fasting state. Therefore, celecoxib $\mathrm{AUC}_{\infty}$ and $T_{1 / 2 \mathrm{el}}$ were not calculated for these subjects and only 30 subjects were included in the statistical analysis of these parameters for celecoxib (Fig. 1). A further subject had a measurable pre-dose $O$-desmethyltramadol concentration before the first treatment period, despite confirming that he/she had abided by protocol instructions on study drug intake. However, the pre-dose $O$-desmethyltramadol concentration was less than $5 \%$ of the $C_{\max }$ value in the first treatment period for this subject and was therefore considered unlikely to influence bioequivalence assessment. This subject was therefore included in the pharmacokinetic analyses.

\subsection{Pharmacokinetics}

Mean tramadol plasma concentration-time profiles were similar in fed and fasting conditions (Fig. 2a). Pharmacokinetic parameters for tramadol are summarised in Table 2. $T_{\text {max }}$ and $T_{1 / 2 \text { el }}$ parameters were significantly different ( $p$ value $<0.05$ ) between fed and fasting states. For $C_{\max }$, although statistically significant differences were found, geometric LS mean fed-to-fasting ratios and their $90 \%$ CIs were within the a

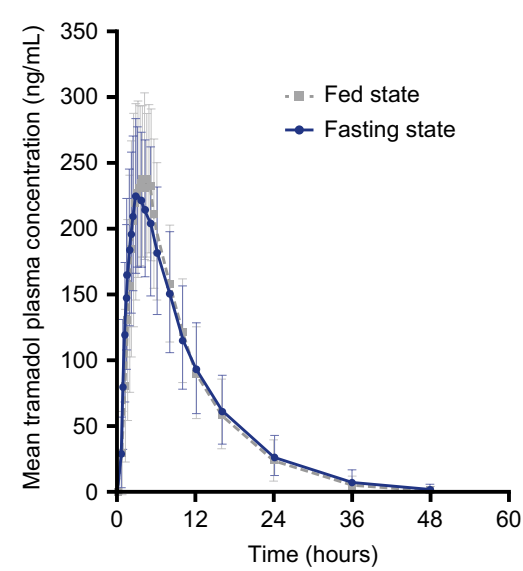

b

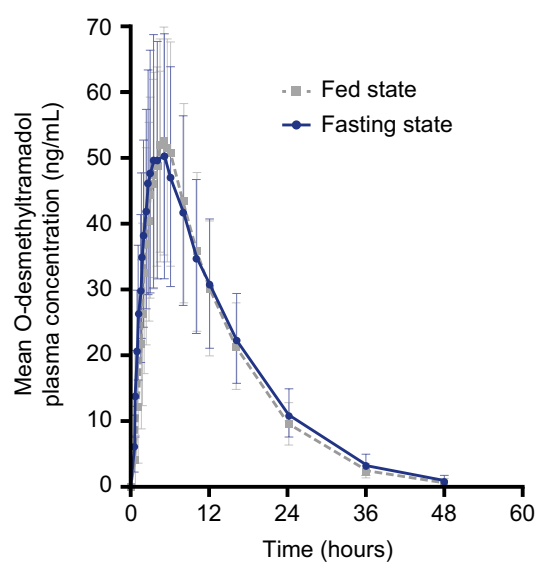

C

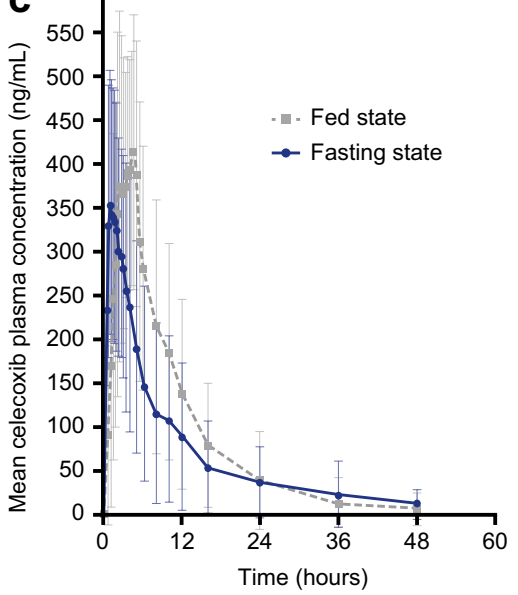

Fig. 2 Mean plasma concentration versus time profiles for tramadol (a); $O$-desmethyltramadol (b); and celecoxib (c) following a single dose of CTC administered under fed or fasting conditions $(n=33)$. Data are means \pm standard deviation. CTC Co-Crystal of Tramadol-Celecoxib 
Table 2 Summary of PK parameters of tramadol, $O$-desmethyltramadol and celecoxib following a single dose of CTC administered under fed or fasting conditions

\begin{tabular}{|c|c|c|c|c|}
\hline \multirow[t]{2}{*}{ Parameter } & \multicolumn{2}{|l|}{$\begin{array}{l}\text { Fed } \\
n=33\end{array}$} & \multicolumn{2}{|l|}{$\begin{array}{l}\text { Fasting } \\
n=33\end{array}$} \\
\hline & Mean & SD & Mean & SD \\
\hline \multicolumn{5}{|l|}{ Tramadol } \\
\hline$C_{\max }(\mathrm{ng} / \mathrm{mL})^{*}$ & 267.02 & 57.4 & 243.61 & 57.0 \\
\hline$T_{\max }(\mathrm{h})^{\mathrm{a}, *}$ & 3.67 & $1.75-5.50$ (range) & 2.67 & $1.50-6.00$ (range) \\
\hline $\mathrm{AUC}_{\mathrm{t}}(\mathrm{ng} \cdot \mathrm{h} / \mathrm{mL})$ & 2719.70 & 911.1 & 2773.15 & 942.9 \\
\hline $\mathrm{AUC}_{\infty}(\mathrm{ng} \cdot \mathrm{h} / \mathrm{mL})$ & 2801.47 & 907.7 & 2857.37 & 948.6 \\
\hline$T_{1 / 2 \mathrm{el}}(\mathrm{h})^{*}$ & 6.11 & 1.52 & 6.58 & 1.5 \\
\hline \multicolumn{5}{|l|}{$O$-desmethyltramadol } \\
\hline$C_{\max }(\mathrm{ng} / \mathrm{mL})$ & 56.26 & 17.6 & 53.14 & 319.3 \\
\hline$T_{\max }(\mathrm{h})^{\mathrm{a}}$ & 4.50 & $2.33-6.00$ (range) & 4.00 & $2.33-8.00$ (range) \\
\hline $\mathrm{AUC}_{t}(\mathrm{ng} \cdot \mathrm{h} / \mathrm{mL})^{*}$ & 763.50 & 210.7 & 802.77 & 230.4 \\
\hline $\mathrm{AUC}_{\infty}(\mathrm{ng} \cdot \mathrm{h} / \mathrm{mL})^{*}$ & 781.47 & 209.4 & 825.04 & 231.8 \\
\hline$T_{1 / 2 \mathrm{el}}(\mathrm{h})^{*}$ & 6.74 & 1.5 & 7.38 & 1.5 \\
\hline \multicolumn{5}{|l|}{ Celecoxib } \\
\hline$C_{\max }(\mathrm{ng} / \mathrm{mL})^{*}$ & 525.57 & 182.4 & 410.31 & 171.1 \\
\hline$T_{\max }(\mathrm{h})^{\mathrm{a}, *}$ & 3.67 & $1.00-6.00$ (range) & 1.25 & $0.75-6.00$ (range) \\
\hline $\mathrm{AUC}_{t}(\mathrm{ng} \cdot \mathrm{h} / \mathrm{mL})^{*}$ & 4411.11 & 2576.1 & 3450.35 & 2508.4 \\
\hline $\mathrm{AUC}_{\infty}(\mathrm{ng} \cdot \mathrm{h} / \mathrm{mL})^{\mathrm{b}, *}$ & 4514.43 & 3002.1 & 3615.20 & 2921.1 \\
\hline$T_{1 / 2 \mathrm{el}}(\mathrm{h})^{\mathrm{b}, *}$ & 8.22 & 3.0 & 11.30 & 4.4 \\
\hline
\end{tabular}

ANOVA analysis of variance, $A U C_{t}$ cumulative area under the concentration-time curve from time zero to time of last observed quantifiable plasma concentration, $A U C_{\infty}$ extrapolated area under the concentration-time curve to infinity, $C_{\max }$ maximum plasma concentration, $C T C$ Co-Crystal of Tramadol-Celecoxib, $P K$ pharmacokinetic, $S D$ standard deviation, $T_{1 / 2 e l}$ elimination half-life, $T_{\max }$ time to maximum plasma concentration

*A statistically significant difference between fed and fasting state $[p<0.05$; ANOVA model, with fixed factors of subject effect (nested within sequence), the treatment received, the period at which it was received and the sequence in which each treatment was received]

${ }^{a}$ Data are median (range)

${ }^{\mathrm{b}} n=30$ for celecoxib $\mathrm{AUC}_{\infty}$ and $T_{1 / 2 \mathrm{el}}$

$80-125 \%$ range, indicating the absence of a food effect for this parameter. In the case of AUC values, no statistically significant differences were found, and the geometric LS mean fed-to-fasting ratios and their $90 \%$ CIs were within the $80-125 \%$ range, indicating the absence of a food effect (Table 3).

Mean plasma concentration-time curves for $O$-desmethyltramadol were also similar in fed and fasting conditions (Fig. 2b). $O$-desmethyltramadol $C_{\max }, T_{\max }$, AUC and $T_{1 / 2 \mathrm{el}}$ values are shown in Table 2 . There was no statistically significant difference in $C_{\max }$ between fed and fasting conditions. Although $O$-desmethyltramadol AUC and $T_{1 / 2 \mathrm{el}}$ values had a $p$ value $<0.05$ between fed and fasting states, the geometric LS mean fed-to-fasting ratios and corresponding $90 \%$ CIs were between 80 and $125 \%$ for $C_{\max }$ and for AUC values, indicating the absence of a food effect for $O$-desmethyltramadol (Table 3).

The pharmacokinetics of celecoxib were subject to a food effect (Fig. 2c). All celecoxib pharmacokinetic parameters (Table 2) showed a significant difference $(p$ value $<0.05$ ) between the fed and fasting states. $T_{\max }$ was significantly delayed under fed versus fasting conditions (median value of 3.67 and $1.25 \mathrm{~h}$, respectively). The half-life of celecoxib was shorter in the fed state than in the fasting state (8.22 and $11.30 \mathrm{~h}$ ). Fed-to-fasting geometric LS mean ratios and corresponding $90 \%$ CIs for $C_{\max }$, [130.91\% (116.98-146.49)], $\mathrm{AUC}_{t}\left[131.71 \%\right.$ (124.54-139.30)] and $\mathrm{AUC}_{\infty}[129.34 \%$ (121.78-137.38)] were outside the 80 and $125 \%$ range for comparative bioavailability, indicating a food effect on celecoxib pharmacokinetics (Table 3 ).

\subsection{Safety}

There were no serious AEs or deaths and no subject was withdrawn from the study for safety reasons. Twenty-three subjects $(23 / 36 ; 63.9 \%)$ experienced a total of 88 AEs. After administration of CTC in fed conditions, 17 subjects (17/36; $47.2 \%$ ) reported $46 \mathrm{AEs}$, of which 43 were assessed as possibly related to study drug. After receipt of CTC in fasting conditions, 20 subjects $(20 / 35 ; 57.1 \%)$ reported 42 AEs, of 
Table 3 Comparative bioavailability of tramadol, $O$-desmethyltrama$\mathrm{dol}$ and celecoxib following a single dose of CTC administered under fed or fasting conditions

\begin{tabular}{lrrrr}
\hline Parameter & \multicolumn{2}{l}{$\begin{array}{l}\text { Geometric LS } \\
\text { means }(n=33)\end{array}$} & Ratio (\%) & $90 \%$ CIs \\
\cline { 2 - 3 } & \multicolumn{1}{c}{ Fed } & Fasting & & \\
\hline Tramadol & & & & \\
$C_{\max }(\mathrm{ng} / \mathrm{mL})$ & 261.05 & 236.40 & 110.43 & $105.34-115.76$ \\
$\mathrm{AUC}_{t}(\mathrm{ng} \cdot \mathrm{h} / \mathrm{mL})$ & 2570.81 & 2616.33 & 98.26 & $94.48-102.20$ \\
$\mathrm{AUC}_{\infty}(\mathrm{ng} \cdot \mathrm{h} / \mathrm{mL})$ & 2660.36 & 2704.35 & 98.37 & $94.85-102.02$ \\
$O$-desmethyltramadol & & & & \\
$C_{\max }(\mathrm{ng} / \mathrm{mL})$ & 53.27 & 49.66 & 107.29 & $101.60-113.3$ \\
$\mathrm{AUC}_{t}(\mathrm{ng} \cdot \mathrm{h} / \mathrm{mL})$ & 733.18 & 768.41 & 95.41 & $92.40-98.53$ \\
$\mathrm{AUC}_{\infty}(\mathrm{ng} \cdot \mathrm{h} / \mathrm{mL})$ & 752.37 & 791.27 & 95.08 & $92.08-98.19$ \\
$\mathrm{Celecoxib}$ & & & & \\
$C_{\max }(\mathrm{ng} / \mathrm{mL})$ & 499.47 & 381.54 & 130.91 & $116.98-146.49$ \\
$\mathrm{AUC}_{t}(\mathrm{ng} \cdot \mathrm{h} / \mathrm{mL})$ & 4037.17 & 3065.13 & 131.71 & $124.54-139.30$ \\
$\mathrm{AUC}_{\infty}(\mathrm{ng} \cdot \mathrm{h} / \mathrm{mL})^{\mathrm{a}}$ & 4087.73 & 3160.35 & 129.34 & $121.78-137.38$ \\
\hline
\end{tabular}

$A U C_{t}$ cumulative area under the concentration-time curve from time zero to time of last observed quantifiable plasma concentration, $A U C$ $\infty$ extrapolated area under the concentration-time curve to infinity, $C I$ confidence interval, $C_{\max }$ maximum plasma concentration, CTC Co-Crystal of Tramadol-Celecoxib, $L S$ least-squares

${ }^{\mathrm{a}} n=30$ for celecoxib $\mathrm{AUC}_{\infty}$

which 40 were assessed as possibly related to study drug. Dizziness [reported in 7/36 (19.4\%) and 9/35 (25.7\%) of subjects in fed and fasting states, respectively], somnolence [8 (22.2\%) and $4(11.4 \%)$ ] and nausea [5 (13.9\%) and 5 (14.3\%)] were the most commonly reported AEs (Table 4). AEs ranged from mild to severe. Four severe AEs were recorded after treatment in the fed state [nausea $(n=2)$ and vomiting $(n=2)]$ and two after treatment in the fasting state [dizziness $(n=1)$ and vomiting $(n=1)$; Table 4]. Two subjects required rescue medication for headache following the first administration of CTC. Two subjects had clinically significant urinalysis laboratory values after the study (one subject was positive for white blood cells and one for red blood cells). Both were lost to follow-up before end-of-study. No clinically significant effects on vital signs, ECGs or physical examination were noted during the study.

\section{Discussion}

In this randomised crossover study of healthy male and female subjects, the bioavailability of tramadol after a single dose of CTC, as determined by bioequivalence analyses, was unaffected by food, whereas that of celecoxib was increased after consumption of a high-fat meal. Absorption of celecoxib was also slowed in the fed state compared with the fasting state. The single-dose safety profile of CTC was
Table 4 Summary of adverse events

\begin{tabular}{|c|c|c|}
\hline & $\begin{array}{l}\text { Fed } \\
n=36\end{array}$ & $\begin{array}{l}\text { Fasting } \\
n=35\end{array}$ \\
\hline Subjects with at least one AE, $n(\%)$ & $17(47.2)$ & $20(57.1)$ \\
\hline Number of AEs & 46 & 42 \\
\hline \multicolumn{3}{|c|}{$\begin{array}{l}\text { Most commonly reported AEs occurring in at least two subjects } \\
\text { (Number of subjects/number of AEs) }\end{array}$} \\
\hline \multicolumn{3}{|l|}{ Nervous system disorders } \\
\hline Dizziness & $7 / 7$ & $9 / 11$ \\
\hline Somnolence & $8 / 9$ & $4 / 4$ \\
\hline Headache & $3 / 4$ & $3 / 3$ \\
\hline Hypoaesthesia & $1 / 5$ & $1 / 1$ \\
\hline \multicolumn{3}{|l|}{ Gastrointestinal disorders } \\
\hline Nausea & $5 / 5$ & $5 / 6$ \\
\hline Vomiting & $2 / 7$ & $2 / 3$ \\
\hline \multicolumn{3}{|c|}{ General disorders and administration site conditions } \\
\hline Fatigue & $0 / 0$ & $2 / 2$ \\
\hline Pallor & $1 / 1$ & $1 / 1$ \\
\hline \multicolumn{3}{|c|}{ Injury, poisoning and procedural complications } \\
\hline Vessel puncture site reaction & $2 / 2$ & $0 / 0$ \\
\hline \multicolumn{3}{|c|}{ Severe AEs (Number of subjects/number of severe AEs) } \\
\hline \multicolumn{3}{|l|}{ Gastrointestinal disorders } \\
\hline Nausea & $2 / 2$ & $0 / 0$ \\
\hline Vomiting & $2 / 2$ & $1 / 1$ \\
\hline \multicolumn{3}{|l|}{ Nervous system disorders } \\
\hline Dizziness & $0 / 0$ & $1 / 1$ \\
\hline
\end{tabular}

Each subject was counted only once per drug administration within each system organ class and MedDRA preferred AE term

$A E$ adverse event, MedDRA medical dictionary for regulatory activities

similar following administration in the fed or fasting state and CTC was well tolerated under both conditions.

The primary results from the current study, namely that food had no effect on tramadol bioavailability but increased celecoxib bioavailability, are consistent with those observed when the standard drug formulations of tramadol and celecoxib are administered individually. Food intake does not affect the bioavailability of standard-formulation tramadol [13]. However, for standard-formulation celecoxib, high-fat meals are known to increase celecoxib $C_{\max }$ by approximately $40-60 \%$ and AUC by $10-20 \%$, and delay absorption by approximately $1-2 \mathrm{~h}$, compared with fasting conditions $[14,15]$. In vitro studies have suggested that this effect of food on celecoxib bioavailability may be linked to an increase in bile secretion [18].

In line with regulatory requirements $[16,17]$, this Phase 1 food effect study was conducted using a randomized, twoperiod, two-sequence, single-dose, crossover design, in healthy volunteers. While such a design has some inherent limitations, we do not believe that these negatively impact on data interpretation. For example, the European Medicines 
Agency recommends single-dose studies for their superior sensitivity in detecting differences in $C_{\max }$ when compared to the same design using multiple doses. In addition, regulatory authorities suggest that bioequivalence studies be conducted in healthy volunteers where possible, and that this permits extrapolation to other populations [16, 17].

The data reported herein are supported by findings from other CTC clinical trials. The fasting pharmacokinetic data obtained in the present study are similar to those collected in earlier Phase 1 studies of CTC, in which dosing was carried out under fasting conditions $[11,12]$. In the present study, median $T_{\max }$ values of celecoxib and tramadol after CTC dosing under fasting conditions were 1.25 and $2.67 \mathrm{~h}$, respectively. In an earlier single-dose Phase 1 CTC study, corresponding median $T_{\max }$ values after CTC dosing under fasting conditions were 1.50 and $2.67 \mathrm{~h}$ for celecoxib and tramadol, respectively. These values were slightly higher in the case of tramadol, and lower in the case of celecoxib, than those obtained after administration of reference products alone or in free combination [11]. In the present study, mean fasting $C_{\max }$ values after CTC administration were 410 and $244 \mathrm{ng} / \mathrm{mL}$, for celecoxib and tramadol, respectively. This compares with values of 313 and $263 \mathrm{ng} / \mathrm{mL}$ in the earlier single-dose Phase 1 study. Similar results were also observed for AUC values [11]. Differences in values between the two studies are within the range of inter-subject variability. The modified pharmacokinetic profile of tramadol and celecoxib after CTC administration, seen in this earlier study, is consistent with the fasting pharmacokinetic data reported here.

In the present study, the single-dose safety profile of CTC was similar in both fed and fasting conditions. CTC was well tolerated and there were no clinically significant effects of treatment on vital signs, ECGs or physical examination, and no serious AEs, safety-related withdrawals or deaths. The total number of AEs and the number of subjects who experienced at least one $\mathrm{AE}$ were similar under both conditions. The most frequently observed AEs were consistent with commonly reported AEs from previous Phase 1 and Phase 2 studies of CTC [10-12] and for standard drug formulations of tramadol and celecoxib $[13,14]$.

\section{Conclusion}

In conclusion, food increased the bioavailability of celecoxib from CTC in healthy subjects but had no effect on the bioavailability of tramadol. These findings are in line with those reported for the standard drug formulations of the individual reference products $[13,14]$. The food effect on the bioavailability of celecoxib after CTC administration was not sufficient to indicate that any dose adjustment would be required. In line with dosing recommendations for other tramadol and celecoxib preparations [14, 19], it is anticipated that no specific dosing recommendations will be necessary in relation to meal times. Following demonstration of efficacy in a Phase 2 study of acute pain following an oral surgical procedure [10], CTC is now under evaluation in Phase 3 studies of bunionectomy with osteotomy [20], acute pain following an oral surgical procedure [21] and hysterectomyrelated pain [22].

Acknowledgements We thank all study participants and study staff. The study was funded by Esteve Pharmaceuticals, S.A. and was carried out by Algorithme Pharma. Bioanalytical analyses were conducted by Esteve Pharmaceuticals, S.A. Pharmacokinetic analyses were conducted by Algorithme Pharma. Medical writing support was provided by Hannah Mace, MPharmacol at Aspire Scientific (Bollington, UK), and was funded by Mundipharma Research GmbH \& Co.KG (Limburg, Germany). CTC is in development by Mundipharma Research and Esteve Pharmaceuticals, S.A., as MR308 and E-58425.

Author contributions GE and SV were involved in the conception and design of the study and the interpretation of data. NG and CP-S were involved in the conception and design of the study. KS was involved in the interpretation of data. MEn, MEs, ML and ES were involved in the acquisition, analysis, and interpretation of data. All authors reviewed drafts of the manuscript, approved the final version and agree to be accountable for the work.

\section{Compliance with Ethical Standards}

Conflict of interest GE, MEn, MEs, NG and CP-S are employees of Esteve Pharmaceuticals, S.A. SV was an employee of Esteve Pharmaceuticals, S.A. at the time of the clinical trial. $\mathrm{KS}$ is an employee of Mundipharma Research Ltd. ES and ML have no potential conflict of interest to declare.

Ethical approval All procedures performed in studies involving human participants were in accordance with the ethical standards of the institutional and/or national research committee and with the 1964 Helsinki declaration and its later amendments or comparable ethical standards.

Informed consent Informed consent was obtained from all individual participants included in the study.

Open Access This article is distributed under the terms of the Creative Commons Attribution-NonCommercial 4.0 International License (http://creativecommons.org/licenses/by-nc/4.0/), which permits any noncommercial use, distribution, and reproduction in any medium, provided you give appropriate credit to the original author(s) and the source, provide a link to the Creative Commons license, and indicate if changes were made.

\section{References}

1. Duggirala NK, Perry ML, Almarsson O, Zaworotko MJ. Pharmaceutical cocrystals: along the path to improved medicines. Chem Commun (Camb). 2016;52(4):640-55.

2. Shan N, Perry ML, Weyna DR, Zaworotko MJ. Impact of pharmaceutical cocrystals: the effects on drug pharmacokinetics. Expert Opin Drug Metab Toxicol. 2014;10(9):1255-71.

3. European Medicines Agency. Reflection paper on the use of cocrystals of active substances in medicinal products. 2015. http:// 
www.ema.europa.eu/docs/en_GB/document_library/Scientific _guideline/2015/07/WC500189927.pdf. Accessed 12 Oct 2017.

4. Almansa C, Mercè R, Tesson N, Farran J, Tomàs J, Plata-Salamán CR. Co-Crystal of Tramadol hydrochloride-Celecoxib (CTC): a novel API-API co-crystal for the treatment of pain. Cryst Growth Des. 2017;17(4):1884-92.

5. Chou R, Gordon DB, de Leon-Casasola OA, Rosenberg JM, Bickler S, Brennan T, et al. Management of postoperative pain: a clinical practice guideline from the American Pain Society, the American Society of Regional Anesthesia and Pain Medicine, and the American Society of Anesthesiologists' Committee on Regional Anesthesia, Executive Committee, and Administrative Council. J Pain. 2016;17(2):131-57.

6. Polomano RC, Fillman M, Giordano NA, Vallerand AH, Nicely KL, Jungquist CR. Multimodal analgesia for acute postoperative and trauma-related pain. Am J Nurs. 2017;117(3 Suppl 1):S12-26.

7. Moore RA, Derry S, Aldington D, Wiffen PJ. Single dose oral analgesics for acute postoperative pain in adults-an overview of Cochrane reviews. Cochrane Database Syst Rev. 2015;9:CD008659.

8. Yaksh TL, Woller SA, Ramachandran R, Sorkin LS. The search for novel analgesics: targets and mechanisms. F1000Prime Rep. 2015;7:56.

9. Merlos M, Portillo-Salido E, Brenchat A, Aubel B, Buxens J, Fisas A, et al. Administration of a co-crystal of tramadol and celecoxib in a 1:1 molecular ratio produces synergistic antinociceptive effects in a postoperative pain model in rats. Eur J Pharmacol. 2018. https://doi.org/10.1016/j.ejphar.2018.06.022.

10. López-Cedrún J, Videla S, Burgueño M, Juárez I, Aboul-Hosn S, Martín-Granizo R, et al. Co-Crystal of Tramadol-Celecoxib in patients with moderate to severe acute post-surgical oral pain: a dose-finding, randomised, double-blind, placebo- and active-controlled, multicentre, phase II trial. Drugs R\&D. 2018;18(2):137-48.

11. Videla S, Lahjou M, Vaqué A, Sust M, Encabo M, Soler L, et al. Single-dose pharmacokinetics of Co-Crystal of Tramadol-Celecoxib: results of a four-way randomized open-label phase I clinical trial in healthy subjects. Br J Clin Pharmacol. 2017;83(12):2718-28.

12. Videla S, Lahjou M, Vaqué A, Sust M, Escriche M, Soler L, et al. Pharmacokinetics of multiple doses of Co-Crystal of
Tramadol-Celecoxib: findings from a 4-way randomized openlabel Phase I clinical trial. Br J Clin Pharmacol. 2018;84(1):64-78.

13. European Medicines Agency. Summary of product characteristics for tramadol hydrochloride $50 \mathrm{mg}$ capsules. 2017. http://www. medicines.org.uk/EMC/medicine/24186/SPC. Accessed 12 Oct 2017.

14. European Medicines Agency. Summary of product characteristics for Celebrex $100 \mathrm{mg}$ capsule. 2016. http://www.medicines.org.uk/ emc/medicine/14534. Accessed 12 Oct 2017.

15. Center for Drug Evaluation and Research. Clinical pharmacology and biopharmaceutics review for Celebrex (celecoxib). 1999. https://www.accessdata.fda.gov/drugsatfda_docs/nda/99/21156 -S007_Celebrex_biopharmr.pdf. Accessed 02 Nov 2017.

16. Food and Drug Administration. Guidance for industry. Food-effect bioavailability and fed bioequivalence studies. 2002. https://www. fda.gov/downloads/drugs/guidancecomplianceregulatoryinforma tion/guidances/ucm070241.pdf. Accessed 06 Nov 2017.

17. European Medicines Agency. Guideline on the investigation of bioequivalence. 2010. http://www.ema.europa.eu/docs/en_GB/ document_library/Scientific_guideline/2010/01/WC50007003 9.pdf. Accessed 11 Jun 2017.

18. Lyng E, Havenaar R, Shastri P, Hetsco L, Vick A, Sagartz J. Increased bioavailability of celecoxib under fed versus fasted conditions is determined by postprandial bile secretion as demonstrated in a dynamic gastrointestinal model. Drug Dev Ind Pharm. 2016;42(8):1334-9.

19. McCormack PL. Celecoxib: a review of its use for symptomatic relief in the treatment of osteoarthritis, rheumatoid arthritis and ankylosing spondylitis. Drugs. 2011;71(18):2457-89.

20. ClinicalTrials.gov. Co-crystal E-58425 vs tramadol and celecoxib for moderate to severe acute pain after bunionectomy. Phase III clinical trial. 2017. https://clinicaltrials.gov/ct2/show/NCT03 108482. Accessed 18 Dec 2017.

21. ClinicalTrials.gov. Efficacy and safety in a randomised acute pain study of MR308 (Tramadol/Celecoxib). (STARDOM1). 2017. https://clinicaltrials.gov/ct2/show/NCT02982161. Accessed 12 Oct 2017.

22. ClinicalTrials.gov. Efficacy and safety in a randomised acute pain study of MR308. (STARDOM2). 2017. https://clinicaltrials.gov/ ct2/show/NCT03062644. Accessed 12 Oct 2017.

\section{Affiliations}

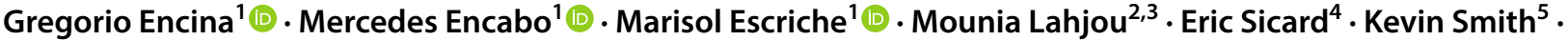 Neus Gascon ${ }^{6}$ - Carlos Plata-Salamán ${ }^{6}$ (D) Sebastián Videla ${ }^{6,7}$}

1 Esteve Pharmaceuticals, S.A., Parc Cientific Barcelona C/ Baldiri Reixac 4-8, 08028 Barcelona, Spain

2 Algorithme Pharma, 575 Armand-Frappier Boulevard, Laval, Quebec H7V 4B3, Canada

3 Present Address: Innovaderm Research Inc., 1851, Sherbrooke Est, Bureau 502, Montreal, Quebec H2K 4L5, Canada

4 Algorithme Pharma, 1200, Beaumont Avenue, Montreal H3P 3P1, Canada
5 Mundipharma Research Ltd, Cambridge Science Park, Milton Road, Cambridge CB4 0GW, UK

6 Esteve Pharmaceuticals, S.A., TORRE ESTEVE, Passeig de la Zona Franca, 109, 08038 Barcelona, Spain

7 Present Address: Clinical Research Support Unit, Clinical Pharmacology Department, Bellvitge University Hospital/IDIBELL, Barcelona, Spain 